\title{
IMBEDDING OF ABELIAN CATEGORIES
}

BY

\author{
SAUL LUBKIN
}

1. Introduction. In this paper, we prove the following

EXACT IMBEDDING THEOREM. Every abelian category (whose objects form a set) admits an additive imbedding into the category of abelian groups which carries exact sequences into exact sequences.

As a consequence of this theorem, every object $A$ of $a$ has "elements"namely, the elements of the image $A^{\prime}$ of $A$ under the imbedding-and all the usual propositions and constructions performed by means of "diagram chasing" may be carried out in an arbitrary abelian category precisely as in the category of abelian groups.

In fact, if we identify $a$ with its image $a^{\prime}$ under the imbedding, then a sequence is exact in $a$ if and only if it is an exact sequence of abelian groups. The kernel, cokernel, image, and coimage of a map $f$ of $a$ are the kernel, cokernel, image, and coimage of $f$ in the category of abelian groups; the map $f$ is an epimorphism, monomorphism, or isomorphism if and only if it has the corresponding property considered as a map of abelian groups. The direct product of finitely many objects of $Q$ is their direct product as abelian groups. If $A \in Q$, then every subobject [4] of $A$ is a subgroup of $A$, and the intersection (or sum) of finitely many subobjects of $A$ is their ordinary intersection (or sum); the direct (or inverse) image of a subobject of $A$ by a map of $a$ is the usual set-theoretic direct (or inverse) image. Moreover, if

$$
A_{0} \underset{f_{0}}{\rightarrow} A_{1} \underset{g_{1}}{\leftarrow} A_{2} \underset{f_{1}}{\rightarrow} A_{3} \underset{g_{2}}{\leftarrow} \cdots \underset{f_{n}}{\rightarrow} A_{2 n+1}
$$

are maps in $a$, and the set-theoretic composite $f_{n} g_{n}^{-1} \cdots g_{1}^{-1} f_{0}$ is a welldefined function $A_{0} \rightarrow A_{2 n+1}$, then this composite is the image of a unique map of $a$, this map of $a$ being independent of the exact imbedding $a \rightarrow \mathcal{E}$ chosen.

In particular, many of the proofs and constructions in [2] remain valid in an abstract abelian category-e.g., the Five Lemma, the construction of connecting homomorphisms, etc.

If the abelian category $a$ is not a set, then each of its objects is represented under the imbedding by a group that need not be a set.

I am very grateful to Professor S. Eilenberg for the encouragement and patience he has shown during the writing of this paper.

2. Exhaustive systems. In $\$ \S 2-4, a$ denotes a fixed set-theoretically legitimate abelian category, and $\mathcal{E}$ the category of abelian groups.

Received by the editors February 11, 1960 and, in revised form, April 8, 1960. 
An exhaustive system of monomorphisms in an abelian category $Q$ is a nonempty direct system $\left(A_{i}, \alpha_{i j}\right) i \in D$, of monomorphisms in $a$ such that

(E) If $i \in D$ and $f: A_{i} \rightarrow B$ is a monomorphism then there exists $j \geqq i$ such that $\alpha_{\imath j}=f$.

Lemma 1. If $\left(A_{i}, \alpha_{i j}\right)$ is an exhaustive system of monomorphisms in a then

(1.1) If $f, f^{\prime}: A^{\prime} \rightarrow A$ are maps in $\mathfrak{Q}$ and $f \neq f^{\prime}$ then there exists $i \in D$ such that $\operatorname{Hom}\left(f, A_{i}\right) \neq \operatorname{Hom}\left(f^{\prime}, A_{i}\right)$.

(1.2) If $f: A \rightarrow B$ is a monomorphism, $i \in D$ and $g: A \rightarrow A_{i}$ a map then there exists $j \geqq i$ in $D$ and $k: B \rightarrow A_{j}$ such that $k f=\alpha_{i j} g$.

Proof. (1.1). Let $i \in D$ and $g: A_{i} \rightarrow A \oplus A_{i}$ be the injection. Then by (E) there exists $j \geqq i$ in $D$ such that $\alpha_{i j}=g$. But then $A_{j}=A \oplus A_{i}$. Let $h: A \rightarrow A_{j}$ be the injection. Then $\operatorname{Hom}\left(f, A_{i}\right)$ and $\operatorname{Hom}\left(f^{\prime}, A_{i}\right)$ disagree on $h \in \operatorname{Hom}\left(A, A_{j}\right)$, so that $\operatorname{Hom}\left(f, A_{j}\right) \neq \operatorname{Hom}\left(f^{\prime}, A_{j}\right)$.

Proof. (1.2). Let $(C, \gamma)$ be the cokernel of the map $A \rightarrow B \oplus A_{i}$ with coordinates $f,-g$.
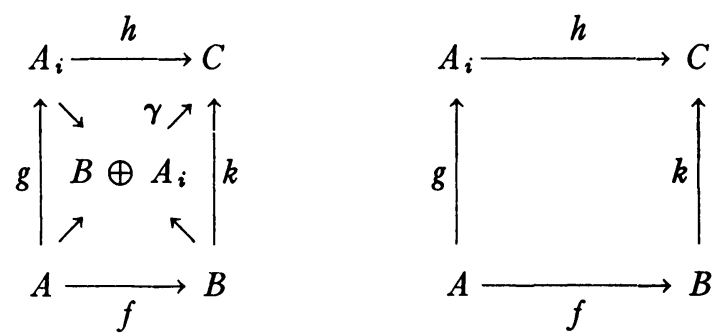

Then if $h$ is the composite $B \rightarrow B \oplus A_{i} \rightarrow C$, and $k$ the composite $A_{i} \rightarrow B \oplus A_{i} \rightarrow C$, we have $k f=h g$, and $k$ is a monomorphism. By (E), there exists $j \geqq i$ such that $\alpha_{i j}=h$. Then $k f=\alpha_{i j} g$, as required. Q.E.D.

3. The basic construction. In this section, we shall prove

THEOREM 1. If $a$ is an abelian category then there exists an exhaustive system of monomorphisms in $a$.

We first obtain some preliminary results.

LEMMA 1. If $\left(A_{i}, \alpha_{i j}\right)_{i, j \in I}$ is a directed system of monomorphisms in $a$, $i_{0} \in I$, and $f: A_{i_{0}} \rightarrow B$ is a monomorphism, then there exists a directed system of monomorphisms $\left(A_{i}, \alpha_{i j}\right)_{i, j \in I^{\prime}}$ extending $\left(A_{i}, \alpha_{i j}\right)_{i, j \in I}$ and $a j \geqq i$ in $I^{\prime}$ such that $\alpha_{i j}=f$.

Proof. Let $J$ be the directed subset of $I$ consisting of all $i \geqq i_{0}$. Let $J^{*}$ be a set equipotent to $J$ and disjoint from $I$, and let $j \rightarrow j^{*}$ be a bijection from $J$ onto $J^{*}$. Let $I^{\prime}=I \cup J^{*}$ be the directed set containing $I$ as a directed subset such that, for $j, k \in J, j^{*} \leqq k^{*}$ iff $j \leqq k$; for $j \in J$ and $i \in I, j^{*} \$ i$, and $i \leqq j^{*}$ iff $i \leqq j$. 
For $j \in J$, let $A_{j^{*}}$ be the cokernel of the map $A_{i_{0}} \rightarrow A_{j} \oplus B$ with coordinates the maps $\alpha_{i_{0} j}: A_{i_{0}} \rightarrow A_{j}$ and $-f: A_{i_{0}} \rightarrow B$. Then we have a commutative diagram of monomorphisms:

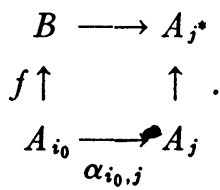

The assignment $j \rightarrow A_{j^{*}}$ is functorial, so that for $j \leqq k$ in $J$, we have a mono-

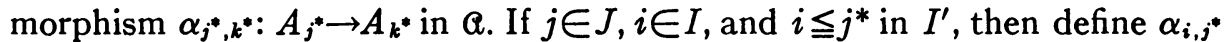
to be the composite $A_{i} \rightarrow^{\alpha_{i j}} A_{j} \rightarrow A_{j^{*}}$. Then $\left(A_{i}, \alpha_{i j}\right)_{i j \in I^{\prime}}$ is a direct family of monomorphisms in $a$ extending $\left(A_{i}, \alpha_{i j}\right)_{i, j \in I}$, and $\alpha_{i_{0}, i_{0}}{ }^{*}=f$. Q.E.D.

LEMma 2. If $\left(A_{i}, \alpha_{i j}\right)_{i, j \in I}$ is a direct system of monomorphisms in $a$, then there exists a direct system $\left(A_{i}, \alpha_{i j}\right)_{i, j \in I^{\prime}}$ of monomorphisms extending $\left(A_{i}, \alpha_{i j}\right)_{i, j \in I}$ such that, whenever $i \in I$ and $f: A_{i} \rightarrow B$ is a monomorphism in $a$, there exists $j \geqq i$ in $I^{\prime}$ with $\alpha_{i j}=f$.

Proof. Let $P$ be the set of all pairs $(k, f)$ where $k \in I$ and $f$ is a monomorphism of $a$ with domain $A_{k}$. Well-order $P$ by a relation $\leqq$. For each $(k, f) \in P$, define a directed family $\left(A_{i}, \alpha_{i j}\right)_{i, j \in I_{(k, f)}}$ of monomorphisms as follows:

Having defined $\left(A_{i}, \alpha_{i j}\right)_{i, j \in I_{\left(k^{\prime}, f^{\prime}\right)}}$ for $\left(k^{\prime}, f^{\prime}\right)<(k, f)$, put $D_{(k, f)}$ $=I \cup \bigcup_{\left(k^{\prime}, f^{\prime}\right)<(k, f)} I_{\left(k^{\prime}, f^{\prime}\right)}$. Then apply Lemma 1 to the family $\left(A_{i}, \alpha_{i j}\right)_{i, j \in D_{(k, f)}}$; let $\left(A_{i}, \alpha_{i j}\right)_{i, j \in I_{(k, S)}}$ be the family obtained.

Put $I^{\prime}=\bigcup_{(k, f) \in P} I_{(k, f)}$. Then the family $\left(A_{i}, \alpha_{i j}\right)_{i, j \in I^{\prime}}$ has the desired properties. Q.E.D.

Proposition 1. If $\left(A_{i}, \alpha_{i j}\right)_{i, j \in D}$ is any direct system of monomorphisms in $a$, then there exists an exhaustive system of monomorphisms $\left(A_{i}, \alpha_{i j}\right)_{i, j \in D^{\prime}}$ extending $\left(A_{i}, \alpha_{i j}\right)_{i, j \in D}$.

Proof. For each non-negative integer $q$, let $\left(A_{i}, \alpha_{i j}\right)_{i, j \in D_{q}}$ be defined inductively by:

$D_{0}=D$. Having defined $\left(A_{i}, \alpha_{i j}\right)_{i, j \in D_{q}}$, let $\left(A_{i}, \alpha_{i j}\right)_{i, j \in D_{Q+1}}$ be the direct system obtained by applying Lemma 2 to $\left(A_{i}, \alpha_{i j}\right)_{i, j \in D_{q}}$.

Putting $D^{\prime}=\cup_{q \geq 0} D_{q}$, we see that $\left(A_{i}, \alpha_{i j}\right)_{i, j \in D^{\prime}}$ is an exhaustive system of monomorphisms. Q.E.D.

Proof of Theorem 1. Let $D$ be a directed set consisting of a single element $i$. Let $A_{i}$ be any object of $Q$, and $\alpha_{i, i}$ be the identity map of $A_{i}$. Then $\left(A_{i}, \alpha_{i j}\right)_{i, j \in D}$ is a directed system of monomorphisms of $a$. Proposition 1 completes the proof.

REMARK. Note that the cardinal number of the indexing set of the exhaustive systems we have constructed is that of the set of monomorphisms of $a$. (This is so even if $a$ is not a set.)

4. The limiting process. Let $\left(A_{i}, \alpha_{i j}\right)$ be an exhaustive system of mono- 
morphisms in the abelian category $a$, indexed by the directed set $D$. Define a functor

$$
F: a^{*} \rightarrow \mathcal{E} \text { by } F(A)=\underset{\longrightarrow_{i}}{\lim } \operatorname{Hom}\left(A, A_{i}\right), \quad F(f)=\underset{\lim _{i}}{\operatorname{Hom}}\left(f ; A_{i}\right) .
$$

Then it is easy to see that $F$, being the direct limit of additive, left-exact functors mapping into $\mathcal{E}$, is itself left-exact and additive.

LEMMA 1. The functor $F$ is an exact imbedding $\left({ }^{1}\right)$ from $a^{*}$ into $\mathcal{E}$.

Proof. If $A \neq A^{\prime}$ in $Q$, then for each $i \in D, \operatorname{Hom}\left(A, A_{i}\right) \cap \operatorname{Hom}\left(A^{\prime}, A_{i}\right)=\varnothing$. Hence, the abelian groups $F(A)$ and $F\left(A^{\prime}\right)$ are disjoint and therefore distinct.

If $f_{1}, f_{2}: A^{\prime} \rightarrow A$ are maps in $Q$, then by (1.1) there exists $i \in D$ such that $\operatorname{Hom}\left(f_{1}, A_{i}\right) \neq \operatorname{Hom}\left(f_{2}, A_{i}\right)$. The canonical maps: $\operatorname{Hom}\left(A, A_{i}\right) \rightarrow F(A)$, $\operatorname{Hom}\left(A^{\prime}, A_{i}\right) \rightarrow F\left(A^{\prime}\right)$, being the direct limit of monomorphisms in the category $\mathcal{E}$, are monomorphisms. Hence, considering the commutative diagrams

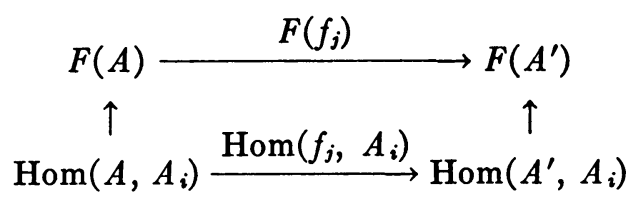

for $j=1,2$, we see that $F\left(f_{1}\right) \neq F\left(f_{2}\right)$. Hence, $F$ is an imbedding.

If $f: B \rightarrow A$ is an epimorphism in $a^{*}$, then $F(f): F(B) \rightarrow F(A)$ is an epimorphism of abelian groups. In fact, if $\bar{g} \in F(A)$, then let $g \in \operatorname{Hom}\left(A, A_{i}\right)$ represent $\bar{g}$. Then $f: A \rightarrow B$ is a monomorphism and $g: A \rightarrow A_{i}$ is a map; choose $j \geqq i$ in $D$ and $k: B \rightarrow A_{j}$ as in (1.2). Then $k f=\alpha_{i j} g$, i.e., $\operatorname{Hom}\left(f, A_{j}\right) k$ $=\operatorname{Hom}\left(A, \alpha_{i j}\right) g$. Let $\bar{k} \in F(B)$ be the element of $F(B)$ represented by $k$. Then chasing elements in the commutative diagram:

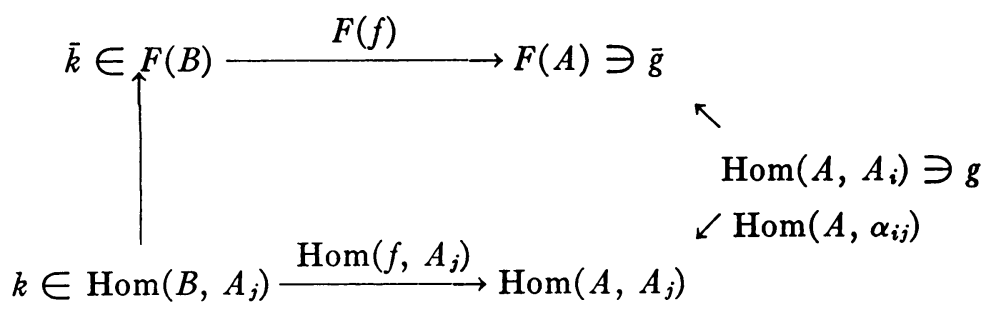

we see that $F(f)(\bar{k})=\bar{g}$, as required.

Since we have already observed that $F$ is left exact, the theorem follows. Q.E.D.

Applying Theorem 1 and Lemma 1 to the dual category $Q^{*}$, we see that $a$ admits an (additive) exact imbedding into $\mathcal{E}$, which proves the Exact Imbedding Theorem.

5. Generalizations. It should be clear that the proof of the Exact Im-

( $\left.{ }^{1}\right)$ Recall that an imbedding from a category $Q$ into a category $B$ is an isomorphism from $Q$ onto a subcategory of $B$. 
bedding Theorem can be extended to arbitrary categories. In this section, we state such a generalization.

If $f, g: A \rightarrow B$ are maps in the category $a$, then the equalizer $E(f, g)$ of $f$ and $g$ (when defined) is the biggest subobject of $A$ on which $f$ and $g$ agree. In an additive category, $E(f, g)=\operatorname{Ker}(f-g), \operatorname{Ker}(f)=E(f, 0)$, so that "equalizer" is a generalization of "kernel" to arbitrary categories.

THEOREM. Let a be a set-theoretically legitimate category closed under direct products of two objects and equalizers of two maps. Then the following three conditions are equivalent:

1. a admits an imbedding into the category of nonempty sets preserving monomorphisms, epimorphisms, equalizers, and finite direct products.

2. a admits an imbedding into the category of nonempty sets preserving epimorphisms, equalizers, and finite direct products.

3. Every diagram:

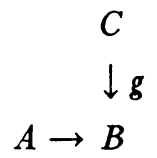

with $g$ an epimorphism can be imbedded in a commutative diagram:

$$
\begin{aligned}
D & \rightarrow C \\
h \downarrow & \\
& \downarrow g \\
A & \rightarrow B
\end{aligned}
$$

with $h$ an epimorphism. Moreover, the canonical $\operatorname{map}, A \times B \rightarrow A$, is an epimorphism.

In addition, if the category $a$ is pointed or additive, then we may assume that the imbeddings in 1 and 2 take values in the category of pointed sets or abelian groups.

6. Element techniques in an abstract abelian category. In this section, we prove the assertions made in $\$ 1$ about exact imbeddings into $\mathcal{E}$. We consider only abelian categories.

It is easy to see that, in an abelian category, if $f=k i$ with $k$ a monomorphism and $i$ an epimorphism, then $k$ is an image and $i$ is a coimage of $f$. This is seen by considering the commutative diagram:

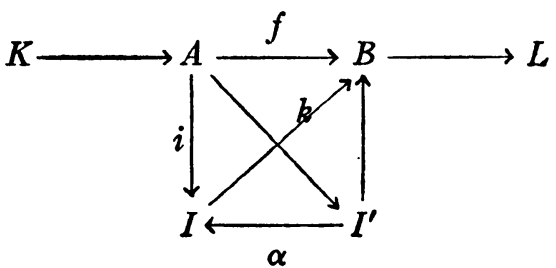


where $K, L$ and $I^{\prime}$ are, respectively, the kernel, cokernel, and image of $f$, and $\alpha$ is uniquely determined.

THEOREм 2. Let $F$ be an exact imbedding from the abelian category $Q$ into the abelian category $B$. Then a sequence (1) $A_{1} \rightarrow^{0} A_{2} \rightarrow A_{3}$ is exact in $Q$ if and only if the image sequence is exact in $B$.

Proof. Necessity is clear; let us prove sufficiency. Suppose that $F\left(A_{1}\right)$ $\rightarrow^{F(g)} F\left(A_{2}\right) \rightarrow^{F(f)} F\left(A_{3}\right)$ is exact in $B$. Then $f g=0$. Hence, if $k=\operatorname{kernel}(f)$ in $Q$, then there exists $i$ such that $g=k i$. But $F(k)=$ kernel $F(f)=$ image $F(g)$ in $B$; since $F(g)=F(k) F(i)$, it follows that $F(i)$ is the coimage of $F(g)$, whence $i$ is an epimorphism in $Q$; clearly $k$ is a monomorphism in $Q$. Since $g=k i$ it follows that $k=\operatorname{image}(g)$, and (1) is exact in Q. Q.E.D.

Henceforth, fixing an exact imbedding into $\varepsilon$ for each abelian category, we use freely the set-theoretic properties of objects and maps in abelian categories.

If $A, B \in Q$, then an (additive) relation $R: A \rightarrow B$ is a subobject $R$ of $A \times B$. If $R: A \rightarrow B$ and $S: B \rightarrow C$ are relations, then their composite $S R$ is the image under the projection $A \times B \times B \times C \rightarrow A \times C$ of $(R \times S) \cap(A \times \Delta \times C)$, where $\Delta$ is the diagonal of $B \times B$. Since this coincides with the set-theoretic definition, composition of relations is associative.

Similarly, one defines the inverse relation $R^{-1}$, and proves that $\left(R^{-1}\right)^{-1}$ $=R$.

If $f: A \rightarrow B$ is a map in $\mathrm{Q}$, then the image of the map $A \rightarrow A \times B$ with coordinates $e_{A}, f$ is the graph $R_{f}$ of $f$; it is the ordinary set-theoretic graph. Hence, $f \rightarrow R_{f}$ is one-to-one and preserves composites. We identify the map $f$ with its graph $R_{f}$.

The relation $R$ is a (graph of some) map if and only if the composite $R \rightarrow A \times B \rightarrow A$ is an isomorphism. Hence, if $F: Q \rightarrow B$ is an exact imbedding of abelian categories, then $R$ is a map if and only if $F(R)$ is a map.

If in the abelian category $Q$,

$$
A_{0} \stackrel{f_{0}}{\rightarrow} A_{1} \stackrel{g_{1}}{\longleftarrow} A_{2} \stackrel{f_{1}}{\rightarrow} A_{3} \stackrel{g_{2}}{\longleftarrow} \ldots \stackrel{f_{n}}{\rightarrow} A_{2 n+1}
$$

are maps and the relation $R_{f_{n}}\left(R_{g_{n}}\right)^{-1} \cdots\left(R_{g_{1}}\right)^{-1} R_{f_{0}}$ is the graph of a map, then this map: $A_{0} \rightarrow A_{2 n+1}$ is called their inverse-composite and is denoted $f_{n} g^{-1} \cdots g_{1}^{-1} f_{0}: A_{0} \rightarrow A_{2 n+1}$.

Using the Exact Imbedding Theorem and Theorem 2, we obtain:

Theorem 3 (Axiomatic Definition of inverse composite). $A$ complete characterization of the inverse-composite $f_{n} g_{n}^{-1} \cdots g_{1}^{-1} f_{0}$ of maps

$$
A_{0} \stackrel{f_{0}}{\rightarrow} A_{1} \stackrel{g_{1}}{\leftarrow} A_{2} \stackrel{f_{1}}{\rightarrow} \ldots \stackrel{f_{n}}{\rightarrow} A_{2 n+1}
$$

in an abelian category is given by: 
(IC1) In the category $\&$ of abelian groups, the inverse-composite $f_{n} g_{n}^{-1} \cdots$ $g_{1}^{-1} f_{0}$ is defined if and only if the corresponding set-theoretic composite is a singlevalued function defined on all of $A_{0}$; in which case the two coincide.

(IC2) If $F: a \rightarrow B$ is an exact imbedding of abelian categories, then the inverse-composite $f_{n} g_{n}^{-1} \cdots g_{1}^{-1} f_{0}$ is defined in $a$ if and only if $F\left(f_{n}\right) F\left(g_{n}\right)^{-1} \ldots$ $F\left(g_{1}\right)^{-1} F\left(f_{0}\right)$ is defined in $B$; in which case, $F\left(f_{n} g_{n}^{-1} \cdots g_{1}^{-1} f_{0}\right)=F\left(f_{n}\right) F\left(g_{n}\right)^{-1}$ ... $F\left(g_{1}\right)^{-1} F\left(f_{0}\right)$.

Combining Theorems 2 and 3, we obtain all the properties of exact imbeddings asserted in the Introduction.

Added in proof (October 25,1960$)$. The theorem of $\$ 5$ can be used to characterize abelian categories:

COROLlARY. If $a$ is an additive category such that kernels, cokernels and finite direct products exist, then $a$ is abelian iff the following three conditions hold:

(1) Every diagram:

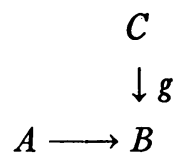

with $g$ an epimorphism can be imbedded in a commutative diagram:

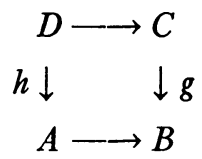

with $h$ an epimorphism.

(2) Every diagram:

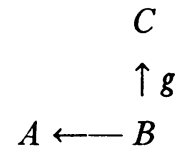

with $g$ a monomorphism can be imbedded in a commutative diagram:

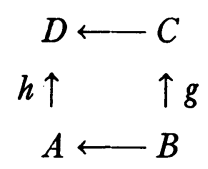

with h a monomorphism.

(3) A map that is both an epimorphism and a monomorphism is an isomorphism.

Proof. In view of (1) and the theorem of $\$ 5, a$ admits an additive imbed$\operatorname{ding} A \rightarrow A^{\prime}, g \rightarrow g^{\prime}$ into $\mathcal{E}$ preserving kernels and epimorphisms, and hence also coimages. Hence, if $f=k z h$ is the canonical factorization of a map $f$ of $a$, 
then $h^{\prime}=\operatorname{Coim}\left(f^{\prime}\right)$ in $\varepsilon$; since $f^{\prime}=k^{\prime} z^{\prime} h^{\prime}$, we must have $k^{\prime} z^{\prime}=\operatorname{Im}\left(f^{\prime}\right)$; whence $k^{\prime} z^{\prime}, z^{\prime}$ and therefore $z$ are monomorphisms in, respectively, $\mathcal{E}, \mathcal{E}$ and $a$. Applying (2) to the dual category $a^{*}$, we see that $z$ is an epimorphism. Hence by (3) $z$ is an isomorphism. Q.E.D.

\section{BIBLIOGRAPHY}

1. D. A. Buchsbaum, Exact categories and duality, Trans. Amer. Math. Soc. vol. 80 (1955) pp. 1-34.

2. H. Cartan and S. Eilenberg, Homological algebra, Princeton University Press, 1956.

3. S. Eilenberg and S. MacLane, General theory of natural equivalences, Trans. Amer. Math. Soc. vol. 58 (1945) pp. 231-294.

4. Kurt Gödel, The consistency of the continuum hypothesis, Annals of Mathematics Studies, no. $3,1940$.

5. A. Grothendieck, Sur quelques points d'algèbre homologique, Tôhoku Math. J. vol. 9 nos. 2-3 (1957) pp. 119-221.

COLUMBia University,

NEW YORK, NEW YoRK 